\title{
Perspective
}

PERSPECTIVE Actualité en histoire de l'art

4 | 2007

Genre et histoire de l'art

\section{Études de genre et histoire de l'art dans le monde germanophone}

\section{Mechthild Fend}

\section{(2) OpenEdition}

1 Journals

\section{Édition électronique}

URL : http://journals.openedition.org/perspective/3582

DOI : 10.4000/perspective.3582

ISSN : 2269-7721

Éditeur

Institut national d'histoire de l'art

\section{Édition imprimée}

Date de publication : 31 décembre 2007

Pagination : 722-730

ISSN : 1777-7852

\section{Référence électronique}

Mechthild Fend, «Études de genre et histoire de l'art dans le monde germanophone », Perspective [En ligne], 4 | 2007, mis en ligne le 31 mars 2018, consulté le 01 octobre 2020. URL : http://

journals.openedition.org/perspective/3582 ; DOI : https://doi.org/10.4000/perspective.3582 


\title{
Études de genre et histoire de l'art dans le monde germanophone
}

\author{
Mechthild Fend
}

En 1977, la Documenta, exposition d'art contemporain la plus importante d'Allemagne, afficha pour la première fois un choix d'artistes explicitement féministes comme Valie Export, Friederike Paetzold et Ulrike Rosenbach. Ulrike Rosenbach présenta la photographie d'un Hercule surdimensionné qui tenait un écran vidéo sous le bras. La vidéo montrait le visage d'une femme qui répétait et sans cesse le mot «femme ». Cette installation vidéo spécialement conçue pour cette manifestation portait le titre Héraclès - Hercule - King Kong. Quant à la photographie, elle se référait à la copie de l'Hercule Farnèse antique placé sur l'édicule octogonal qui couronne la grande cascade du parc du château de Wilhelmshöhe, un des symboles de la ville de Kassel ${ }^{1}$. Héros grec et modèle identificatoire des souverains, Hercule incarne ici la force et le pouvoir patriarcaux contre lesquels l'artiste prend inlassablement la parole, la différence dans l'échelle des représentations exprimant l'idée des rapports de pouvoir inégaux entre hommes et femmes. Dans le contexte précis de la Documenta, cette différence apparaît comme un symbole de l'intervention de femmes-artistes face à des institutions d'art dominées par les hommes.

\section{Les années 1970 : Frauen in der Kunst}

2 Dès la fin des années 1960 et directement en lien avec le Neue Frauenbewegung [jeune mouvement féministe], des artistes comme Valie Export s'étaient consacrées, parallèlement à leurs collègues américaines, aux thèmes du corps et de la sexualité à travers des performances, des créations photographiques, cinématographiques et vidéo, ainsi que des textes théoriques ${ }^{2}$. Aux États-Unis apparurent quasiment au même moment des historiennes de l'art féministes, dont Linda Nochlin, qui posa la questionclef de l'histoire de l'art féministe avec son article « Why Have There Been No Great Women Artists? » de $1970^{3}$. De son côté, l'Allemagne dut attendre le milieu des années 1970 avant de voir des historiennes et critiques d'art agir dans une approche féministe. 
3 Au début, l'intérêt portait avant tout sur les femmes-artistes contemporaines, ainsi que sur les œuvres de femmes-artistes des siècles passés, dont la plupart étaient à redécouvrir et à resituer. C'était d'abord des expositions qui questionnaient le rôle des femmes dans l'art ${ }^{4}$. Dans le cadre du Neuer Berliner Kunstverein [Nouvelle association d'art berlinoise], le groupe Frauen in der Kunst [Femmes dans l'art] organisa l'exposition Künstlerinnen International 1877-1977 [Femmes-artistes International 1877-1977] qui se tint en 1977 au château de Charlottenburg de Berlin. De même, les premières publications en histoire de l'art féministe étaient principalement consacrées aux femmes-artistes. L'ouvrage de Renate Berger - issu de sa thèse de doctorat soutenue à Hambourg, Malerinnen auf dem Weg ins 20. Jahrhundert. Kunstgeschichte als Sozialgeschichte [Les femmes-peintres à l'aube du $\mathrm{xx}^{\mathrm{e}}$ siècle. L'histoire de l'art en tant qu'histoire sociale] - interrogeait les œuvres et les conditions de travail et d'apprentissage des femmes-artistes en Allemagne au tournant du siècle ${ }^{5}$. Écrite dans une perspective féministe, l'étude suivait la méthode de l'histoire sociale de l'art et examinait le contexte social et institutionnel qui avait marqué le travail des femmesartistes.

4 L'ouvrage en deux volumes Frauen in der Kunst [Les femmes dans l'art], paru en 1980 sous la direction de la journaliste Gislind Nabakowski, du théoricien d'art Peter Gorsen et de la cinéaste Helke Sander, était particulièrement consacré à l'art et au film contemporains. La réunion des trois auteurs illustrait à quel point théorie et pratique étaient imbriquées. Les volumes contiennent un grand nombre de contributions de femmes-artistes et de réalisatrices qui témoignent de l'avance de plusieurs années qu'avaient certaines artistes, comme Valie Export, de par rapport à leurs collègues en histoire de l'art, par leurs travaux théoriques sur l'art féministe et sur la créativité féminine. Les ouvrages intègrent également des traductions d'articles de Laura Mulvey («Visual Pleasure and Narrative Cinema» de 1973-1975) et de Caroline Sheldon («Lesbians and Film» de 1977) et rendent compte ainsi, de manière assez précoce, d'une orientation lesbienne. Cette publication chercha par ailleurs à se confronter aux approches anglo-américaines de l'histoire de l'art et des film studies féministes. Les années suivantes, la réception en Allemagne des travaux d'historiennes de l'art, du cinéma et de la littérature anglosaxonne, tout comme l'échange direct avec les collègues des pays anglosaxons, continua de stimuler l'intérêt des gender studies dans l'histoire de l'art allemande.

\section{Le rôle des congrès des historiennes de l'art}

5 Depuis les années 1980, l'histoire de l'histoire de l'art féministe peut être parfaitement récapitulée à travers une manifestation unique dans les pays germanophones ${ }^{6}:$ le Kunsthistorikerinnen-Tagung [Congrès des historiennes de l'art] qui a connu sept réunions à intervalles irréguliers entre 1982 et 2002. Les publications issues de ces congrès donnent un large aperçu de l'évolution des questions de genre dans l'histoire de l'art germanophone qui, dans un premier temps, était tournée principalement vers les recherches féministes sur la femme (feministische Frauenforschung). Elles sont ici présentées selon les thématiques traitées, tout en suivant la chronologie générale.

6 Les congrès se sont déroulés dans différentes villes en Allemagne, en Autriche et en Suisse. Chacun a été organisé par un groupe spécifique d'historiennes de l'art, occupant pour la plupart des postes de chercheurs de durée déterminée, ainsi que par des 
doctorantes et des étudiantes. Contrairement aux États-Unis, où les colloques de la College Art Association disposaient dès les années 1970 de sections féministes ${ }^{7}$, l'Allemagne ne s'est ouverte qu'en 1990 - à l'occasion du XXII Congrès des historiens de l'art - à l'histoire de l'art féministe dans une séance plénière qui est par ailleurs restée unique dans son format programmatique. Cette position à part de l'histoire de l'art féministe est aussi liée à l'histoire de l'association professionnelle des historiens de l'art allemands. À partir de 1968, et avec les mutations sociopolitiques qui ont suivi, l'aile gauche, progressiste, qui avait fondé en 1968 le Ulmer Verein [Association d'Ulm], s'est séparée de la tendance conservatrice de l'institution, regroupée dans le Verband deutscher Kunsthistoriker [VDK, Association des historiens de l'art allemands]. Les historiennes de l'art qui ont commencé au milieu des années 1970 à formuler des interventions féministes au sein de la discipline étaient plutôt proches du Ulmer Verein qui n'organisait cependant pas de grands congrès annuels, mais des colloques thématiques plus réduits. En 1988, cette association a fondé une section consacrée à la recherche sur la femme en histoire de l'art (Frauenforschung in der Kunstgeschichte) qui se compose de plusieurs groupes de travail ${ }^{8}$. Cette alliance n'a pas toujours été sans difficultés car, dans le contexte des années post-68, les discussions avec les adeptes d'une histoire de l'art marxiste portaient essentiellement, en Allemagne et dans d'autres pays, sur l'importance du genre en tant que catégorie sociale ${ }^{9}:$ l'appartenance $^{\prime}$ au genre masculin ou féminin devait-elle être considérée comme un facteur d'inégalité sociale aussi important ou non que celui de "classe "? Malgré ces différences, le premier colloque féminin Frau - Kunst - Gesellschaft - kritische Wissenschaft [Femme - Art - Société - Études critiques] a eu lieu en 1982 à Marbourg, sous l'égide du Ulmer Verein.

Outre la formulation d'une perspective critique face à une science dite " universelle » mais tout aussi assujettie à la domination masculine, ce colloque s'est proposé de développer un questionnement différent de la discipline et de ses objets d'études. Les contributions recueillies dans l'ouvrage collectif Frauen, Kunst, Geschichte [Femmes, art, histoire] de 1984 brassent des thématiques larges, allant du Moyen Âge au xx siècle. Abordant la peinture, la sculpture, l'architecture et la photographie, elles interrogent aussi bien le rôle des femmes-artistes que la représentation de la femme dans les arts ${ }^{10}$.

8 Entre autres approches méthodologiques apparaît surtout le motif d'une critique féministe qui passe au crible les paradigmes «objectivité » et "rationalisme » de la recherche traditionnelle. Il semblait également évident qu'il fût nécessaire de se réunir d'abord sans les collègues masculins - pratique toujours en vigueur, du moins pour ce qui concerne les intervenantes au congrès des historiennes de l'art - même si cela fut critiqué par la suite ${ }^{11}$. Malgré le succès croissant de ces réunions (jusqu'à huit cents participantes), les interventions internationales et les liens de plus en plus importants tissés avec l'histoire de l'art universitaire, ils ont su garder une distance critique face à la discipline établie. Grâce à leur régularité et leur fréquence (initialement tous les deux ans) et à la présence assidue des participantes et des intervenantes, certains sujets et problématiques ont bénéficié d'un débat approfondi, ce qui me semble être une particularité des études de genre dans l'histoire de l'art allemande.

\section{Le corps morcelé}

9 Une des discussions les plus importantes portait sur les représentations de la violence contre les femmes et sur la violence structurelle des images - débat dont l'actualité 
politique était confirmée par les campagnes et les luttes anti-pornographiques des années 1970 et 1980. Le déclencheur de cette discussion avait été une communication de Renate Berger lors du congrès de 1985, qui s'était interrogée sur le nu féminin et notamment sur la représentation de corps féminins morcelés dans l'art du $\mathrm{xx}^{\mathrm{e}}$ siècle ${ }^{12}$. Ce faisant, elle partait de la distinction établie par John Berger (reprenant et inverssant Kenneth Clark) entre "nude " (définie comme une mise en scène visuelle qui fait du corps féminin un objet du regard masculin) et "naked» (état naturel de l'être-en-soi). Son texte défendait plus généralement un point de vue contre la réification du corps féminin représenté. Renate Berger s'intéressait avant tout aux représentations des corps féminins fragmentés, telles que les figurations expressionnistes des meurtres par plaisir (Lustmorde), les montages surréalistes et les performances des Actionnistes viennois. Comme cela a été souligné dans le débat qui a suivi sa communication, l'auteure avait confondu les actes de violence réels avec leur représentation visuelle. Ainsi, répondant directement à Renate Berger, Sigrid Schade critiquait le «mythe du corps entier " qu'elle avait perçu dans sa critique des corps morcelés en tant que concept bourgeois totalisant ${ }^{13}$. Sigrid Schade s'appuyait sur des réflexions poststructuralistes et psychanalytiques (lacaniennes en particulier) selon lesquelles le corps entier ne serait qu'une construction imaginaire toujours liée au phantasme du corps morcelé. Des représentations de corps apparemment entiers et idéaux sont fondées sur des processus sélectifs issus eux-mêmes de fragmentations, comme en témoignent de manière impressionnante des processus de création, par exemple chez Dürer. Sigrid Schade mettait en avant les œuvres d'artistes surréalistes, comme celles de Hans Bellmer, en tant que critiques des représentations cliché de la féminité1 ${ }^{14}$. Reste cependant les effets que ces représentations peuvent susciter chez les spectateurs féminins et masculins, qui empêchent de garder une position distancée face à des images de scènes violentes.

C'est en ce sens que Kathrin Hoffmann-Curtius intervenait dans le débat, proposant de discuter la question de la violence sous un autre angle. Dans le contexte des tableaux représentant des meurtres par plaisir (Lustmorde) chez les artistes expressionistes, elle insistait, tout en faisant la distinction entre représentation et scène représentée, sur les "croisements entre une action criminelle et un acte artistique ", ainsi que sur la violence structurale à l'œuvre dans chaque «transformation » d'un modèle vivant en œuvre d'art ${ }^{15}$. Les travaux de Julia Kristeva, de Teresa de Lauretis et d'Elisabeth Bronfen constituaient les bases théoriques de ce propos, selon lequel la transformation du corps en image est nécessairement violente ${ }^{16}$. C'est lors du congrès en 1988, dont l'un des axes directeurs était la représentation de la violence ${ }^{17}$, Kathrin Hoffmann-Curtius avait déjà, pour la première fois, présenté ces hypothèses. La discussion avait porté particulièrement sur les effets violents des systèmes de représentation et partait de l'idée qu'un corps naturel, libre de tout système, n'existe pas. Par conséquent, féminité et masculinité ne devaient plus être liés à l'anatomie des sujets, mais compris en tant que positions symboliques qu'ils incarnent. Les communications s'intéressaient à la structure du système de représentation dominant, à l'association entre femme et image ${ }^{18}$ et à la violence de la représentation par rapport à la dimension réflexive des médias, notamment de morceaux de corps qui sont montrés dans des films ${ }^{19}$.

11 Les théories postmodernes ou poststructuralistes sur la représentation, ainsi que des approches similaires issues de la théorie française et des film studies américaines, étaient accueillies et approfondies surtout à travers ce débat sur la violence ${ }^{20}$. 
L'exposition viennoise de 1985, Kunst mit Eigensinn [L'art obstiné], sous le commissariat de Silvia Eiblmayer, marqua dans ce contexte un événement également important : elle se focalisait sur le postmodernisme en présentant des œuvres de femmes-artistes contemporaines, européennes et américaines ${ }^{21}$ explorant des thèmes de l'identités, du corps fragmenté, des média, etc. Le catalogue contient notamment une version en allemand du texte «Discourse of Others. Feminism and Postmodernism» de Craig Owen ${ }^{22}$.

\section{Critique de la masculinité en histoire de l'art}

Les mythes de la masculinité et particulièrement celui de l'artiste occupaient un autre champ thématique important. Dès les premiers travaux sur des femmes-artistes, notamment celui de Renate Berger, il était clair que la recherche féministe ne se restreignait pas uniquement au rôle des femmes (même si elle se nommait encore souvent comme telle - « Frauenforschung " [recherches sur la femme]). Il ne s'agissait pas seulement d'ajouter des contributions féminines à une histoire de l'art par ailleurs intouchée, mais aussi d'analyser et de critiquer les structures et institutions responsables de l'exclusion des femmes ${ }^{23}$. Parmi celles-ci comptent aussi les catégories - nullement neutres par rapport aux sexes - d'« artiste » et de " génie », ainsi que le genre de la monographie, à travers lequel l'histoire de l'art participe à la construction de ces figures. Le troisième congrès, qui eut lieu en 1986 à Vienne, interrogeait les représentations et les mythes masculins au même titre que les représentations et les mythes féminins ; ce faisant, il ne tarda pas à initier la recherche sur la masculinité (Männlichkeitsforschung) en histoire de l'art. Comme l'expliquent les auteures, c'est la recherche sur la féminité qui permit « une discussion critique, non-affirmative et nonidentificatoire de l'image masculine et de la mise en scène de l'homme lui-même dans l'art $\|^{24}$.

Le quatrième congrès, celui de Berlin en 1988, déjà mentionné, thématisait à son tour ces constructions sous le titre de "Spiegelungen. Identifikationsmuster patriarchaler Kunstgeschichte » [Jeux de miroir. Les schémas identificatoires d'une histoire de l'art patriarcale $]^{25}$. Les sujets présentés s'intéressaient aussi bien aux représentations de la masculinité et de l'homosexualité (Alexandra Pätzold) qu'aux identités et mises en scènes d'artistes féminins et masculins, comme notamment "Les configurations de la masculinité et de l'autorité » («Er selbst. Konfigurationen von Männlichkeit und Autorität in der deutschen Moderne », p. 21-40) dans les autoportraits de la modernité en Allemagne (Irit Rogoff) ou la reprise de mythes créateurs, tels Pygmalion, dans l'Allemagne postfasciste (Silke Wenk, «Pygmalions Wahlverwandtshaften», p. 59-83). En 1996, une section du sixième congrès reformulait cette question: "Comment s'exprime la différence des sexes dans les conceptions de la créativité, du rôle de l'auteur et de l'œuvre, et de quelle manière ces différences manipulent-elles l'intégration ou l'exclusion de femmes-artistes? ? ${ }^{26}$ ("Wie die Geschlechterdifferenz in Konzeptionen von Kreativität, Autorschaft und Werk eingeschrieben wurde und wie sie den Ein- und Ausschluss von Künstlerinnen reguliert»). Par ailleurs, les mythes de femmes-artistes faisaient l'objet de certaines contributions, dont celle de Reinhild Feldhaus qui examinait les topiques ontologisantes de «naissance » et de «mort » très présentes dans nombre de biographies sur les femmes-artistes ${ }^{27}$. 


\section{Différences sexuelles et différences culturelles}

de l'art féministe. Comme l'ont rappelé les directrices des actes du colloque, «l'élargissement du spectre méthodologique par des approches relevant de la théorie du discours et de la psychanalyse et/ou des procédés déconstructifs » avait déclenché des débats virulents lors d'un précédent colloque à Berlin ${ }^{28}$. La conclusion selon laquelle la féminité serait une construction culturelle et historique, et donc problématique en tant que «facteur identificatoire » (identitätsstiftender Faktor), attirait l'attention sur $"$ ce qu'était l'intention et la base des études féministes ${ }^{29}$. Afin de structurer la discussion et d'éviter la scission idéologique, les congressistes essayèrent de chercher un point d'ancrage historiographique et de définir, à partir d'un examen critique des écrits d'Aby Warburg, leurs propres approches théoriques et méthodologiques. Warburg ne fut pas seulement choisi parce qu'il avait été redécouvert vers 1990 dans l'histoire de l'art germanophone et particulièrement hambourgeoise, mais surtout parce que ses travaux permettaient de créer des liens avec un certain nombre de théories actuelles, dont la Kritische Theorie, développée par Marcuse et Adorno, entre autres, et la psychanalyse, ainsi qu'avec les sujets d'archives et de mémoire, surtout liés aux approches post-structuralistes. Les résultats de cette expérience ont donné lieu à des contributions sur les liens entre la notion de Pathosformel chez Warburg et le discours psychiatrique autour de 1900, en particulier les travaux de Charcot sur l'hystérie (Sigrid Schade), ainsi que sur les rapports entre la Ninfa de Warburg et le texte sur la Gradiva de Freud.

Lors de la première section du sixième congrès de 1995 à Trèves, les réflexions portaient pour la première fois majoritairement sur les liens entre différence sexuelle et différence culturelle. Ce faisant, il s'agissait de thématiser des stratégies théoriques et politiques du post-colonialisme qui avaient déjà été examinées beaucoup plus tôt par la recherche anglo-américaine. Dans sa préface, Viktoria Schmidt-Linsenhoff exprimait ainsi une certaine autocritique en soulignant que l'histoire de l'art féministe germanophone avait trop longtemps ignoré ces problématiques et, en se concentrant sur les intérêts de femmes blanches d'Europe de l'Ouest ou de l'Amérique du Nord, qu'elle était restée attachée aux universalismes qu'elle avait reprochés à la recherche masculine ${ }^{30}$. En incluant des catégories comme "race» et "ethnicité», les gender studies se sont diversifiées. Partant des hypothèses de Judith Butler - son livre Gender Trouble, paru en 1991 aux États-Unis, avait été traduit dès 1992 en allemand ${ }^{31}$-, qui avaient aussitôt suscité de fortes polémiques, les actes du colloque plaidaient pour une compréhension performative des genres et pour une approche de l'identité sexuelle dans ses contextes divers "comme l'orientation sexuelle, l'âge, la classe sociale, la nationalité, l'ethnie, la religion, la couleur de peau, etc. $»^{32}$. Les communications se consacrèrent aux questions de l'orientalisation de la violence (exemple de La mort de Sardanapale de Delacroix, par Kathrin Hoffmann-Curtius), de l'exotisme surréaliste (Gabriele Werner) ou de la mise en scène de la whiteness ["blanchitude »] au cinéma (Ewa Warth). À l'exemple de la figure de la « belle Juive », Hilla Frübis se demandait si l'analyse critique des clichés n'allait pas former à son tour, à travers ses catégorisations, des stéréotypes ${ }^{33}$. D'autres textes contrecarrent le gendering féministe traditionnel des rapports sujet-objet, comme celui de Ruth Noack examinant l'œuvre photographique Nigga' Luvva de Mike Sales, artiste noir britannique qui s'était lui- 
même intéressé aux photographies des Noubas soudanais réalisées par Leni Riefenstahl dans les années 1970. De même, le chapitre "Femmes-artistes " se consacrait au thème de l'identité ethnique, avec les articles d'Irene Below, de Christina Threuter et d'Angela Rosenthal qui confrontaient les œuvres de femmes-artistes d'origine africaine et vivant en Europe, Liz Crossley, Lubaina Himid, Maud Sulter. Leurs créations évoquent les problématiques du racisme, du sexisme, de la migration et de l'exil.

Après ce sixième congrès, le département d'histoire de l'art de l'université de Trèves est resté un centre important pour les études postcoloniales, ce qui est, pour l'histoire de l'art en Allemagne - avec ou sans approche gender - une exception. Depuis 1997 et jusqu'à la fondation en 2005 du Centrum für Postcolonial und Gender Studies [Centre des postcolonial et gender studies], Viktoria Schmidt-Linsenhoff a initié plusieurs projets de recherche interdisciplinaires sur l'interculturalité et sur la différence des genres ${ }^{34}$.

\section{Média et cyberspace}

17 La dernière Kunsthistorikerinnen-Tagung, à l'automne 2002, à l'université Humboldt de Berlin, avait pour thème le genre et les médias. Si l'intention du congrès et de sa publication était de "réagir à la constitution globale de nouvelles techniques et de pratiques de la production d'images et à ses discours environnants ${ }^{35}$, les recherches présentées ont également inclus - sous la perspective de la médialité - les pratiques artistiques plus anciennes comme la peinture, l'architecture, le livre et l'enluminure. $\mathrm{Au}$ centre des discussions se trouvaient les liens entre corps et médias, la compréhension du corps comme médium et/ou du médium comme corps qui peut posséder, notamment dans le cas du livre médiéval, des connotations féminines (texte de Silke Tammen). D'autres axes centraux étaient surtout constitués par les nouveaux médias : photographie, film, vidéo et internet.

Avec la place croissante accordée à l'espace virtuel (cyberspace), l'attention se portait particulièrement sur l'art virtuel et sur le "cyber-féminisme». La question était de savoir si l'espace virtuel est vraiment ce lieu qui permet la transformation à volonté des identités sexuelles afin de neutraliser finalement les différences de genre. Les contributions opéraient des distinctions entre ces positions utopiques en examinant en premier lieu les codes et les symboles sexuels à l'œuvre dans l'espace virtuel. La figure symbolique particulièrement visible est celle de la codification féminine de l'espace virtuel lui-même, dans la droite ligne de la féminisation traditionnelle de l'espace pictural grâce à la perspective centrale. Les cyber-féministes participent par ailleurs à une telle codification féminine de l'espace virtuel lorsqu'elles se réfèrent de manière ontologique à la « matrice féminine » du web ${ }^{36}$. Grâce au choix du thème des médias, les historiennes de l'art ont commencé à participer aux débats, aujourd'hui omniprésents en Allemagne, autour du terme même d'«image» et de l'expression "science de l'image» (Bildwissenschaft). Dans son article «Bildandropologie? Kritik einer Theorie des Visuellen " [L'anthro/andro-pologie de l'image ? Critique d'une théorie du visuel], Hanne Loreck attire l'attention sur les revendications hégémoniales nées du postulat $\mathrm{du}$ «pictorial term». Elle critique également «l'anthropologie de l'image » de Hans Belting, qui dissimule les différences par son approche universaliste des termes de corps et d'image et qui, suivant des idées déconstructivistes et poststructuralistes, occulte aussi les recherches de l'histoire de l'art féministe sur le corps, les médias et l'image ${ }^{37}$. 


\section{De l'utilité d'une revue}

19 germanophones est la revue Frauen, Kunst, Wissenschaft [Femmes, Art, Savoir] qui vient de fêter son vingtième anniversaire et s'appelle dès lors $F K W$ // Frauen für Geschlechterforschung und visuelle Kultur [Femmes pour études de genre et les cultures visuelles]. La revue bianuelle été fondée suite à la troisième KunsthistorikerinnenTagung à Vienne et avait, depuis sa fondation, l'objectif d'établir une certaine continuité dans les débats critiques autour des problèmes spécifiques de la question du genre en histoire de l'art et à permettre une meilleure mise en réseau des historiennes de l'art germanophones ${ }^{38}$. De façon saisissante, les numéros, en particulier ces dernières années, illustrent l'éventail des problématiques et des approches théoriques et méthodologiques. Ils tiennent compte des questionnements postcoloniaux (comme dans l'avant- dernier numéro sous la direction de Marianne Koos Körperfarbe Hautdiskurse. Ethnizität \& Gender in den medialen Techniken der Gegenwartskunst ([Couleur de peau - des discours sur la peau. Ethnicité et Gender dans les techniques médiales de l'art contemporain], $\mathrm{n}^{\circ} 43$, juin 2007) et, sous le titre de Alternative Körper ([Corps alternatifs], $n^{\circ} 29$, juin 2000), du dépassement des frontières des genres, en incorporant des impulsions méthodologiques venant des queer studies et des transgender studies qui s'opposent aux catégories identitaires figées. Le numéro consacré aux héros (Helden, $\mathrm{n}^{\circ}$ 41, juin 2006) s'inscrit cependant plutôt dans une recherche critique sur la masculinité.

méros précédents se sont intéressés aux phénomènes politico-culturels tels que la culture de la mémoire et la patrie sous l'angle des genres. Ce qui avait commencé dans les années 1980 sous la forme des recherches sur la femme (Frauenforschung) a pris ici la forme d'une approche orientée selon les questions de genre qui s'intéressent à la féminité, à la masculinité et à la transsexualité, tout en admettant aussi les controverses entre les différentes intentions politiques des gender studies féministes, gays-lesbiennes ou queer.

\section{Un champ de recherche novateur mais fragile institutionnellement}

21 Surtout lorsque les études de genre en histoire de l'art sont présentées d'une manière très résumée, comme je l'ai fait ici, il en ressort une vision pertinente de la richesse et de l'originalité des travaux menés, de la variété des sujets et des approches méthodologiques. Ces études peuvent revendiquer le fait qu'elles ont été les premières à introduire des perspectives poststructuralistes, postmodernes et postcoloniales dans l'histoire de l'art germanophone. Ce bilan de leur succès ne doit pourtant pas faire croire qu'elles seraient désormais incorporées dans le canon de la discipline ou reconnues comme une des méthodes principalement suivies. Le problème est plus complexe. En particulier, dans les années 1990, certaines universités et leurs ministères de tutelle ont reconnu que l'on ne pouvait pas complètement renoncer à un champ de recherche aussi important et novateur, et quelques collègues femmes, parmi les pionnières de l'histoire de l'art féministe, ont occupé des chaires spécifiquement consacrées à la recherche sur la femme et sur le genre (parmi lesquelles Sigrid Schade à Brême, Viktoria Schmidt-Linsenhoff à Trèves et Silke Wenk à Oldenburg). Depuis ces

Perspective, 4 | 2007 
dix dernières années, plusieurs universités, dont l'université Humboldt de Berlin et l'université Albert-Ludwig de Fribourg, ont instauré des cursus interdisciplinaires pour les gender studies auxquels contribuent des collègues de différentes disciplines. Les études de genre en histoire de l'art peuvent aujourd'hui être menées à bien dans des contextes de recherche variés, d'autant plus que quelques collègues-hommes ont aussi commencé à intégrer la notion de genre dans leurs recherches, même si la plupart ne tiennent pas compte de la tradition féministe de cette approche. Pourtant, dans nombre d'universités, il est encore possible de faire des études d'histoire de l'art sans croiser - et encore moins se familiariser avec - un seul discours critique féministe ou la catégorie de genre.

Il reste à voir si ces postes de professeures dans le champ de la recherche sur la femme et sur le genre pourront garder leur orientation thématique au moment où leurs titulaires atteindront l'âge de la retraite. La suppression de ces postes serait, à mon avis, hautement regrettable puisque ce sont ces postes et ces professeures qui ont contribué à donner un lieu aux gender studies, à diriger nombre de thèses dans ce domaine et à générer, en lien avec des réseaux assez informels, un panorama de la recherche en histoire de l'art d'un point de vue féministe.

\section{NOTES}

1. Sur cette installation vidéo, voir l'article de Barbara Paul, « Mythos Mann. Ulrike Rosenbachs Videoinstallation 'Herakles - Herkules - King Kong' (1977)», dans Marburger Jahrbuch für Kunstgeschichte, 25, 1998, p. 199-220.

2. Voir surtout, parmi les publications récentes sur Valie Export, Valie Export. Mediale Anagramme, (cat. expo., Berlin, Neue Gesellschaft für Bildende Kunst [NGBK], 2003), Berlin, 2003, et en langue française Valie Export, Caroline Bourgeois éd., (cat. expo., Paris, Centre national de la photographie, 2003), Montreuil, 2003.

3. Linda Nochlin, «Why Have There Been No Great Women Artists? », dans Art News, 1970, 69/9, p. 22-39 et 62-71; trad. fr.: «Pourquoi n'y a-t-il pas eu de grands artistes femmes?», dans Femmes, art et pouvoir et autres essais, Nîmes, 1993, p. 201-244.

4. Künstlerinnen International 1877-1977, Ursula Bierther éd., (cat. expo., Berlin, château de Charlottenburg, 1977), Berlin, 1977.

5. Renate Berger, Malerinnen auf dem Weg ins 20. Jahrhundert. Kunstgeschichte als Sozialgeschichte, Cologne, 1982.

6. J'entends par là l'Allemagne, l'Autriche et la Suisse que je traite ensemble puisque ces pays poursuivent des échanges continus dans le domaine de l'histoire de l'art féministe.

7. Le Women's Caucus for Art a été fondé en 1972 dans le contexte de la College Art Association. En 1989 s'y est ajouté le Queer Caucus for Art: The Lesbian, Gay, Bisexual, and Transgender Caucus for Art, Artists, and Historians.

8. Voir le site internet de la Ulmer Verein : http://www.ulmer.verein.de, et celui de la section de la recherche sur la femme (Frauenforschung): www.ulmer-verein.de/uv/index.php? page $=$ content/AGFrauenforschung.php (12/01/2008). L'organe de diffusion de l'association, les 
Kritische Berichte, publie depuis la fin des années 1980 des numéros thématiques axés sur l'approche du genre.

9. Voir Hilla Frübis, «Kunstgeschichte », dans Christina von Braun, Inge Stephan éd., GenderStudien. Eine Einführung, Stuttgart/Weimar, 2000, p. 262-275.

10. Cordula Bischoff et al. éd., Frauen, Kunst, Geschichte. Zur Korrektur des herrschenden Blicks, Giessen, 1984.

11. Lors du dernier congrès à Berlin, l'appel à contribution a été ouvert et la sélection des participants, anonyme, mais aucun candidat masculin ne s'est présenté.

12. Renate Berger, «Pars pro toto. Zum Verhältnis von künstlerischer Freiheit und sexueller Integrität ", dans Renate Berger, Daniela Hammer-Tugendhat éd., Der Garten der Lüste. Zur Deutung des Erotischen und Sexuellen bei Künstlern und ihren Interpreten, Cologne, 1985, p. 150-199.

13. Troisième congrès des historiennes de l'art à Vienne: Sigrid Schade, «Der Mythos des 'ganzen Körpers'. Das Fragmentarische in der Kunst des 20. Jahrhunderts als Dekonstruktion bürgerlicher Totalitätskonzepte ", dans Ilsebill Barta-Fliedl et alii éd., Frauen, Bilder, Männer, Mythen: kunsthistorische Beiträge, Berlin, 1987, p. 239-260.

14. Sigrid Schade suit ici entre autres les idées de Xavière Gauthier, Surréalisme et sexualité, Paris, 1971.

15. Voir à ce sujet Im Blickfeld: George Grosz «John der Frauenmörder», (cat. expo., Hambourg, Kunsthalle, 1993), Hambourg, 1993 - une exposition petite mais explosive.

16. Im Blickfeld..., cité n. 15, p. 12. Voir entre autres Julia Kristeva, Teresa de Lauretis, «The Violence of Rhetoric: Considerations on Representation and Gender ", dans Semiotica, 54, 1985, p. 11-31, et Elisabeth Bronfen, Over her Dead Body. Death, femininity and the aesthetic, Manchester, 1992.

17. Voir les articles recueillis sous la partie IV de la publication : « Gewaltbilder: Zur ästhetischen Organisation von Macht - Sexualität - Gewalt ", dans Ines Lindner et al. éd., Blickwechsel. Konstruktionen von Männlichkeit und Weiblichkeit in Kunst und Kunstgeschichte, Berlin, 1989, p. 333-499.

18. Silvia Eiblmayr, «Gewalt am Bild - Gewalt am Bild. Zur Inszenierung des weiblichen Körpers in der Kunst des 20. Jahrhunderts ", dans Lindner et al., 1989, cité n. 17, p. 337-357. Voir aussi S. Eiblmayr, Die Frau als Bild. Der weibliche Körper in der Kunst des 20. Jahrhunderts, Berlin, 1993.

19. Katharina Sykora, "Verletzung - Schnitt - Verschönerung ", dans Lindner et al., 1989, cité n. 17, p. 359-367.

20. Voir aussi l'article d'Elisabeth Bronfen, spécialiste en lettres et cultural studies : «Weiblichkeit und Repräsentation - aus der Perspektive von Semiotik, Ästhetik und Psychoanalyse », dans Hadumod Bußmann, Renate Hof éd., Genus: Zur Geschlechterdifferenz in den Kulturwissenschaften, Stuttgart, 1995, p. 408-445.

21. Kunst mit Eigen-Sinn. Aktuelle Kunst von Frauen. Texte und Dokumentation, Silvia Eiblmayr, Valie Export, Monika Prischl-Maier éd., (cat. expo., Vienne, Schweitzergarten, 1985), Vienne, 1985.

22. Publié pour la première fois dans Hal Foster éd., The Anti-Aesthetic. Essays on Postmodern Culture, Washington, 1983.

23. Voir à ce sujet aussi Roszika Parker, Griselda Pollock, Old Mistresses. Art and Ideology, Londres, 1981.

24. "Eine kritische und nicht affirmative oder identifikatorische Auseinandersetzung mit dem Männerbild und mit der Selbstinszenierung des Mannes in der Kunst», Ilsebill Barta, « Vorwort », dans Barta-Fliedl et al., 1987, cité n. 13, p. 9.

25. Lindner et al., 1989, p. 17-107.

26. Le sixième Kunsthistorikerinnen-Tagung s'est déroulé en trois sections différentes qui ont été publiées séparément: pour la première (Trèves), voir Annegret Friedrich, Birgit Haehnel, Viktoria Schmidt-Linsenhoff, Christina Threuter éd., Projektionen. Rassismus und Sexismus in der Visuellen Kultur, Marbourg, 1997 ; pour la deuxième (Tübingen), voir Kathrin Hoffmann-Curtius, 
Silke Wenk éd., Mythen von Autorschaft und Weiblichkeit im 20. Jahrhundert, Marbourg, 1997, p. 7. La troisième section s'est consacrée aux codifications sexuelles au sein de l'artisanat: Cordula Bischoff, Christina Threuter éd., Um-Ordnung. Angewandte Künste und Geschlecht in der Moderne, Marbourg, 1999.

27. Voir à ce sujet aussi la thèse de doctorat de Maike Christadler, Kreativität und Geschlecht : Giorgio Vasaris 'Vite' und Sofonisba Anguissolas Selbst-Bilder, Berlin, 2000.

28. «Die Erweiterung des methodischen Spektrums um diskurstheoretische psychoanalytische Ansätze und/oder dekonstruktive Verfahren hatte ", dans Silvia Baumgart et al. éd., Denkräume zwischen Kunst und Wissenschaft, Berlin, 1993, p. 11.

29. «[...] was das Anliegen und die Grundlage feministischer Wissenschaft sei ».

30. Viktoria Schmidt-Linsenhoff, "Einleitung ", dans Annegret Friedrich et al., Projektionen. Rassismus und Sexismus in der Visuellen Kultur, Marbourg, 1997, p 8-14.

31. Judith Butler, Gender Trouble: feminism and the subversion of identity, New York/Londres, 1990 ; trad. fr. Trouble dans le genre: pour un féminisme de la subversion, Paris, 2005. Sur les débats des thèses de la philosophe américaine en Allemagne, voir la revue de sociologie Feministische Studien « Kritik der Kategorie Geschlecht », 2, 1993.

32. « Aspekte wie sexuelle Orientierung, Alter, Klasse, Nation, Ethos, Religion, Hautfarbe etc. ». 33. L'article «Die Schöne Jüdin. Bilder vom Eigenen und vom Fremden », dans Friedrich et al., 1997, cité n. 29, p. 112-130, est notamment une critique de Carol Ockman, « Two large eyebrows à l'orientale: Ethnic stereotyping in Ingres's Baronne de Rothschild », dans Art History, 14, 1991, p. 521-539.

34. Concernant les nombreuses publications, parues dans le cadre de ces projets de recherche, voir le site du centre : www.uni-trier.de/index.php?id=11692\&L=0\&0= (12/01/2008).

35. «Globale Formierung neuer Techniken und Praktiken der Bildgenerierung und die sie flankierenden Diskurse zu reagieren ».

36. Voir l'introduction de Susanne von Falkenhausen dans Susanne von Falkenhausen et al. éd., Medien der Kunst. Geschlecht, Metapher, Code, Marbourg, 2004, p. 10, ainsi que les articles du chapitre «Geschlechtermetamorphosen des Cyberspace », p. 27-68. Voir aussi Linda Hentschel, Pornotopische Techniken des Betrachtens. Raumwahrnehmung und Geschlechterordnung in visuellen Apparaten der Moderne, Marbourg, 2001.

37. Hans Belting, Bild-Anthropologie, Munich, 2002 ; trad. fr.: Pour une anthropologie des images, Paris, 2004.

38. Voir aussi le site : www.frauenkunstwissenschaft.de/index.php (12/01/2008).

\section{INDEX}

Index géographique : Allemagne

Mots-clés : exposition, femmes-artistes, corps, sexualité, histoire de l'art féministe, histoire de l'art allemand, historiennes de l'art, méthodologie, historiographie

Keywords : exhibition, women artists, body, sexuality, feminist art history, German art history, women art historian, methodology, historiography, gender studies

Index chronologique : 1900, 2000 
AUTEUR

MECHTHILD FEND

University College London 